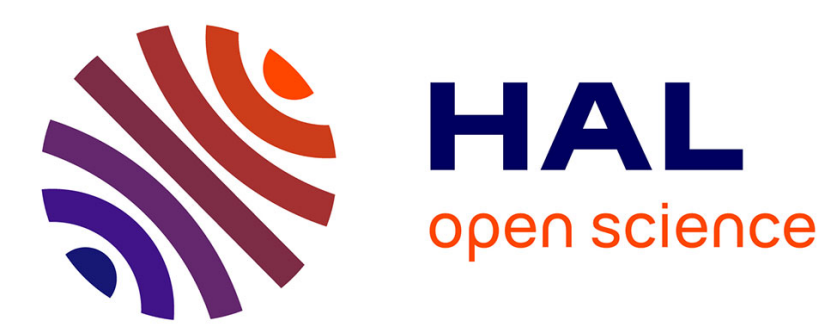

\title{
A new link between the descent algebra of type B, domino tableaux and Chow's quasisymmetric functions
}

Ekaterina A. Vassilieva, Alina Mayorova

\section{To cite this version:}

Ekaterina A. Vassilieva, Alina Mayorova. A new link between the descent algebra of type B, domino tableaux and Chow's quasisymmetric functions. Discrete Mathematics, 2019, 342 (6), pp.1658-1673. 10.1016/j.disc.2019.01.021 . hal-02410964

\section{HAL Id: hal-02410964 https://hal.science/hal-02410964}

Submitted on 14 Dec 2019

HAL is a multi-disciplinary open access archive for the deposit and dissemination of scientific research documents, whether they are published or not. The documents may come from teaching and research institutions in France or abroad, or from public or private research centers.
L'archive ouverte pluridisciplinaire HAL, est destinée au dépôt et à la diffusion de documents scientifiques de niveau recherche, publiés ou non, émanant des établissements d'enseignement et de recherche français ou étrangers, des laboratoires publics ou privés. 


\title{
A new link between the descent algebra of type B, domino tableaux and Chow's quasisymmetric functions
}

\author{
Ekaterina A. Vassilieva ${ }^{\mathrm{a}}$, Alina R. Mayorova ${ }^{\mathrm{a}, \mathrm{b}}$ \\ ${ }^{a}$ Laboratoire d'Informatique de l'Ecole Polytechnique, 91128 Palaiseau Cedex, France \\ ${ }^{b}$ Department of Higher Algebra, Moscow State University, Moscow, Russia
}

\begin{abstract}
Introduced by Solomon in his 1976 paper, the descent algebra of a finite Coxeter group received significant attention over the past decades. As proved by Gessel, in the case of the symmetric group its structure constants give the comultiplication table for the fundamental basis of quasisymmetric functions. We show that this latter property actually implies several well known relations linked to the Robinson-Schensted-Knuth correspondence and some of its generalisations. This provides a new link between these results and the theory of quasisymmetric functions and allows to derive more advanced formulas involving Kronecker coefficients. Using the theory of type B quasisymmetric functions introduced by Chow, we extend this connection to the hyperoctahedral group and derive new formulas relating the structure constants of the descent algebra of type B, the numbers of domino tableaux of given descent set and the Kronecker coefficients of the hyperoctahedral group.
\end{abstract}

Keywords: descent algebra, type B quasisymmetric functions, Kronecker coefficients, RSK-correspondence, hyperoctahedral group, domino tableaux.

\section{Introduction}

For any positive integer $n$ write $[n]=\{1, \cdots, n\}, S_{n}$ the symmetric group on $[n]$ and $i d_{n}$ the identity permutation of $S_{n}$. A composition $\alpha \vDash n$ is a sequence of positive integers $\alpha=\left(\alpha_{1}, \cdots, \alpha_{p}\right)$ such that $\alpha_{1}+\alpha_{2}+\cdots=n$. A composition $\lambda$ of $n$ with its parts sorted in decreasing order is called a partition and denoted $\lambda \vdash n$ or $|\lambda|=n$. A partition $\lambda$ is usually represented as a Young diagram of $n=|\lambda|$ boxes arranged in $\ell(\lambda)$ left justified rows so that the $i$-th row from the top contains $\lambda_{i}$ boxes. A standard Young tableau $T$ is a Young diagram whose boxes are filled with the elements of $[n]$ such that the entries are strictly increasing along the rows and down the columns. The partition given by the number of boxes in each row is its shape and denoted shape $(T)$.

Email addresses: ekaterina.vassilieva@lix.polytechnique.fr (Ekaterina A. Vassilieva), alina.r.m@yandex.ru (Alina R. Mayorova) 
Example 1.1. The following diagrams are standard Young tableaux of shape $\lambda=(6,4,2,1,1)$.
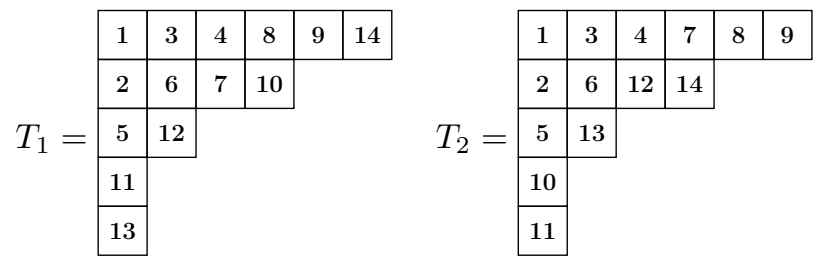

Compositions of $n$ are in natural bijection with subsets of $[n-1]$. For $\alpha \vDash n$ and $I=\left\{i_{1}, i_{2}, \cdots, i_{m}\right\}$ a subset of $[n-1]$ such that $i_{1}<i_{2}<\cdots i_{m}$, we denote $\operatorname{Des}(\alpha)=\left\{\alpha_{1}, \alpha_{1}+\alpha_{2}, \cdots, \alpha_{1}+\alpha_{2}+\cdots \alpha_{p-1}\right\}$ and $\operatorname{comp}(I)=$ $\left(i_{1}, i_{2}-i_{1}, \cdots, i_{m}-i_{m-1}, n-i_{m}\right)$ this bijection and its inverse. As a result the number of compositions of $n$ is $2^{n-1}$.

One important feature of a permutation $\pi$ of $S_{n}$ is its descent set $\operatorname{Des}(\pi)=$ $\{1 \leq i \leq n-1 \mid \pi(i)>\pi(i+1)\}$. Similarly define the descent set of a standard Young tableau $T$ as the subset of $[n-1]$ defined by $\operatorname{Des}(T)=\{1 \leq$ $i \leq n-1 \mid i$ is in a strictly higher row than $i+1\}$. For instance the descent set of the tableaux in Example 1.1 is $\{1,4,9,10,12\}$. We denote $d_{\lambda I}$ the number of standard Young tableaux of shape $\lambda$ and descent set $I$.

Let $D_{I}$ (resp. $B_{I}$ ) be the element of the algebra $\mathbb{C} S_{n}$ defined as the formal sum of all the permutations $\pi$ such that $\operatorname{Des}(\pi)=I$ (resp. $\operatorname{Des}(\pi) \subseteq I)$. In the more general context of finite Coxeter groups, Solomon showed in [19] that the $D_{I}$ 's (resp. $B_{I}$ 's) generate a subalgebra of $\mathbb{C} S_{n}$ of dimension $2^{n-1}$ usually referred to as the Solomon's descent algebra. More specifically, he showed that there exist (non negative integer valued) structure constants $\left(a_{I J}^{K}\right)_{I, J, K \subseteq[n-1]}$ and $\left(b_{I J}^{K}\right)_{I, J, K \subseteq[n-1]}$ that verify:

$$
D_{I} D_{J}=\sum_{K \subseteq[n-1]} a_{I J}^{K} D_{K}, \quad B_{I} B_{J}=\sum_{K \subseteq[n-1]} b_{I J}^{K} B_{K} .
$$

As a result the number of ways to write a fixed permutation $\pi$ of $D_{K}$ as the ordered product of two permutations $\pi=\sigma \tau$ such that $\sigma \in D_{I}$ and $\tau \in D_{J}$ depends only on $\operatorname{Des}(\pi)=K$ and is equal to $a_{I J}^{K}$. If we require instead $\sigma$ and $\tau$ to be respectively in $B_{I}$ and $B_{J}$ then the number of such products is $\sum_{K^{\prime} \supseteq K} b_{I J}^{K^{\prime}}$.

Remark 1.2. The two families of structure constants are linked through the formulas

$$
\sum_{I^{\prime} \subseteq I, J^{\prime} \subseteq J} a_{I^{\prime} J^{\prime}}^{K}=\sum_{K^{\prime} \supseteq K} b_{I J}^{K^{\prime}}
$$

Because of its important combinatorial and algebraic properties the descent algebra received significant attention afterwards. In particular, Garsia and Reutenauer in [11] provide a decomposition of its multiplicative structure and a new combinatorial interpretation of the coefficients $b_{I J}^{K}$ in terms of the 
number of non-negative integer matrices with specified constraints. Bergeron and Bergeron give analogous results in [4] when the symmetric group is replaced by the hyperoctahedral group (Coxeter group of type B). As shown by Norton in [16], the descent algebra of a finite Coxeter group is also strongly related to its 0 -Hecke algebra. In particular, she proves that the dimension of each left principal indecomposable module of the 0-Hecke algebra is equal to the cardinality of the analogue of one the $D_{I}$ 's. She further shows that the analogues of the $a_{I J}^{\emptyset}$ are the entries of the Cartan matrix giving the number of times each irreducible module is a composition factor of each indecomposable module. In the specific case of the symmetric group $S_{n}$, Carter in [6] uses the celebrated Robison-Schensted ( $R S$ ) correspondence to explain the following relation obtained by computation of the Cartan matrix

$$
a_{I J}^{\emptyset}=\sum_{\lambda \vdash n} d_{\lambda I} d_{\lambda J}
$$

Finally, Solomon's descent algebra is dual to the Hopf algebra of quasisymmetric functions (see Section 2) introduced by Gessel in [12]. In particular, he shows that the comultiplication table for their fundamental basis is given by the $a_{I J}^{K}$ 's. In Section 2, we show that Equation (2) and its generalisations are a direct consequence of Gessel's result. Then we use the theory of quasisymmetric functions of type B introduced by Chow ([7]) in Section 3 to provide new analogous formulas in the case of the hyperoctahedral group. Section 4 provides additional results involving more general coefficients.

\section{Descent algebra of the symmetric group}

\subsection{Quasisymmetric functions}

Let $X=\left\{x_{1}, x_{2}, \cdots\right\}$ be an alphabet of commutative indeterminates and $I \subseteq[n-1]$. A quasisymmetric function is a bounded degree formal power series in $\mathbb{C}[X]$ such that for any composition $\left(\alpha_{1}, \cdots \alpha_{p}\right)$ and any strictly increasing sequence of distinct indices $i_{1}<i_{2}<\cdots<i_{p}$ the coefficient of $x_{1}^{\alpha_{1}} x_{2}^{\alpha_{2}} \cdots x_{p}^{\alpha_{p}}$ is equal to the coefficient of $x_{i_{1}}^{\alpha_{1}} x_{i_{2}}^{\alpha_{2}} \cdots x_{i_{p}}^{\alpha_{p}}$. Quasisymmetric functions admit the monomial and fundamental quasisymmetric functions as classical bases:

$$
M_{I}(X)=\sum_{\substack{i_{1} \leq \cdots \leq i_{n} \\ k \in I \Leftrightarrow i_{k}<i_{k+1}}} x_{i_{1}} x_{i_{2}} \cdots x_{i_{n}}, \quad F_{I}(X)=\sum_{\substack{i_{1} \leq \cdots \leq i_{n} \\ k \in I \Rightarrow i_{k}<i_{k+1}}} x_{i_{1}} x_{i_{2}} \cdots x_{i_{n}} .
$$

These two bases are related through

$$
F_{I}(X)=\sum_{I \subseteq J \subseteq[n-1]} M_{J}(X)
$$

Remark 2.1. In Gessel's paper [12], quasisymmetric functions are indexed by compositions and not subsets. However, the bijection between subsets and compositions given in Section 1 makes the definition equivalent. 
For $\lambda \vdash n$ denote also $p_{\lambda}(X)$ and $s_{\lambda}(X)$ the power sum and Schur symmetric functions in the alphabet $X$ indexed by $\lambda$. In what follows, we may remove the reference to $X$ when there is no confusion.

A semistandard Young tableau is a Young diagram whose entries are strictly increasing down the column and non-decreasing along the rows. A tableau has weight $\mu=\left(\mu_{1}, \mu_{2}, \cdots\right)$ if it has $\mu_{i}$ entries equal to $i$. Denote $K_{\lambda \mu}$ the number of semistandard Young tableaux of shape $\lambda$ and weight $\mu$. The decomposition of Schur symmetric functions in the fundamental (e.g. [20, 7.19.7]) and the monomial bases is

$$
\begin{aligned}
s_{\lambda}(X) & =\sum_{I \subseteq[n-1]} d_{\lambda I} F_{I}(X) \\
& =\sum_{I \subseteq[n-1]} K_{\lambda \operatorname{comp}(I)} M_{I}(X) .
\end{aligned}
$$

\subsection{Gessel's relation and its consequences}

For two commutative sets of variables $X=\left\{x_{1}, x_{2}, \cdots, x_{i}, \cdots\right\}$ and $Y=$ $\left\{y_{1}, y_{2}, \cdots, y_{i}, \cdots\right\}$, we denote $X Y$ the set of indeterminates $\left\{x_{i} y_{j} ; x_{i} \in X, y_{j} \in\right.$ $Y\}$ ordered by the lexicographical order. Gessel shows in [12, Thm. 11] that for any subset $K \subseteq[n-1]$

$$
F_{K}(X Y)=\sum_{I, J \subseteq[n-1]} a_{I J}^{K} F_{I}(X) F_{J}(Y) .
$$

We can say that $F_{K}(X Y)$ is the generating series for the coefficients $a_{I J}^{K}$. As stated in introduction, Equation (2) and some of its generalisations are a direct consequence of Equation (6). More precisely, denote by $\chi^{\lambda}$ the irreducible character of $S_{n}$ indexed by partition $\lambda$ and for any $\lambda, \mu, \nu \vdash n$ define the Kronecker coefficients $g(\lambda, \mu, \nu)=1 / n ! \sum_{\omega \in S_{n}} \chi^{\lambda}(\omega) \chi^{\mu}(\omega) \chi^{\nu}(\omega)$. We have the following theorem.

Theorem 2.2. For $I, J, K \subset[n-1]$ let $a_{I, J}^{\emptyset}$ be the structure constant defined in introduction and let $a_{I, J, K}^{\emptyset}=\left[D_{\emptyset}\right] D_{I} D_{J} D_{K}$ be the number of triples of permutations $\sigma_{1}, \sigma_{2}, \sigma_{3}$ of $S_{n}$ such that $\operatorname{Des}\left(\sigma_{1}\right)=I, \operatorname{Des}\left(\sigma_{2}\right)=J, \operatorname{Des}\left(\sigma_{3}\right)=K$ and $\sigma_{1} \sigma_{2} \sigma_{3}=i d_{n}$. Equation (6) directly implies

$$
\begin{aligned}
a_{I J}^{\emptyset} & =\sum_{\lambda \vdash n} d_{\lambda I} d_{\lambda J}, \\
a_{I, J, K}^{\emptyset} & =\sum_{\lambda, \mu, \nu \vdash n} g(\lambda, \mu, \nu) d_{\lambda I} d_{\mu J} d_{\nu K} .
\end{aligned}
$$

Proof. According to Equation (4) $F_{\emptyset}=s_{(n)}$. Then using the Cauchy identity for Schur functions

$$
s_{(n)}(X Y)=\sum_{\lambda \vdash n} s_{\lambda}(X) s_{\lambda}(Y)
$$


and applying Equation (4) once again one gets:

$$
\begin{aligned}
\sum_{I, J \subseteq[n-1]} a_{I J}^{\emptyset} F_{I}(X) F_{J}(Y) & =F_{\emptyset}(X Y)=s_{(n)}(X Y) \\
& =\sum_{\lambda \vdash n} s_{\lambda}(X) s_{\lambda}(Y) \\
& =\sum_{\substack{\lambda \vdash n \\
I, J \subseteq[n-1]}} d_{\lambda I} d_{\lambda J} F_{I}(X) F_{J}(Y) .
\end{aligned}
$$

This can be generalised to prove Equation (8) by noticing that the structure constants verify $a_{I, J, K}^{\emptyset}=\sum_{L} a_{I, L}^{\emptyset} a_{J, K}^{L}$. As a result, Equation (6) extends to

$$
F_{\emptyset}(X Y Z)=\sum_{I, J, K} a_{I, J, K}^{\emptyset} F_{I}(X) F_{J}(Y) F_{K}(Z) .
$$

Finally use the generalised version of Equation (9)

$$
s_{\nu}(X Y)=\sum_{\lambda, \mu} g(\lambda, \mu, \nu) s_{\lambda}(X) s_{\mu}(Y)
$$

to decompose $s_{(n)}(X Y Z)=F_{\emptyset}(X Y Z)$.

Remark 2.3. Denote $\lambda^{\prime}$ the partition corresponding to the transposed Young diagram of $\lambda$. For $I, J \subseteq[n-1]$, the numbers $a_{I J}^{[n-1]}$ are given by

$$
a_{I J}^{[n-1]}=\sum_{\lambda \vdash n} d_{\lambda I} d_{\lambda^{\prime} J}
$$

Proof. According to Equation (4) $F_{[n-1]}=s_{\left(1^{n}\right)}$. Then use the identity for Schur functions

$$
s_{\left(1^{n}\right)}(X Y)=\sum_{\lambda \vdash n} s_{\lambda}(X) s_{\lambda^{\prime}}(Y)
$$

and apply Equation (4) to $s_{\lambda}$ and $s_{\lambda^{\prime}}$.

\subsection{Extension to the RSK-correspondence}

Generalising the RS-correspondence to matrices with non-negative integral entries, Knuth ([14]) proved that for $r, c \vDash n$ the number $m_{r, c}$ of such matrices with row and column sums equal respectively to $r$ and $c$ is given by

$$
m_{r, c}=\sum_{\lambda \vdash n} K_{\lambda r} K_{\lambda c} .
$$

We have the following corollary to Theorem 2.2. 
Corollary 2.4. Equation (12) is also a consequence of Equation (6). Furthermore let $p, q$ and $r$ be three compositions of $n$ and denote $m_{p, q, r}$ the number of three-dimensional arrays $M=\left(M_{i, j, k}\right)$ with non-negative integer entries such that $p_{k}=\sum_{i, j} M_{i, j, k}, q_{j}=\sum_{i, k} M_{i, j, k}$ and $r_{i}=\sum_{j, k} M_{i, j, k}$. Equation (6) implies

$$
m_{p, q, r}=\sum_{\lambda, \mu, \nu \vdash n} g(\lambda, \mu, \nu) K_{\lambda p} K_{\mu q} K_{\nu r} .
$$

Proof. We proceed with the proof of Equation (12).

Denote $A_{I, J}^{K}=\sum_{I^{\prime} \subseteq I, J^{\prime} \subseteq J} a_{I^{\prime}, J^{\prime}}^{K}$. According to Equation (3)

$$
\begin{aligned}
\sum_{I^{\prime}, J^{\prime} \subseteq[n-1]} a_{I^{\prime} J^{\prime}}^{K} F_{I^{\prime}}(X) F_{J^{\prime}}(Y) & =\sum_{I^{\prime}, J^{\prime} \subseteq[n-1]} a_{I^{\prime} J^{\prime}}^{K} \sum_{I \supseteq I^{\prime}, J \supseteq J^{\prime}} M_{I}(X) M_{J}(Y) \\
& =\sum_{I, J \subseteq[n-1]} M_{I}(X) M_{J}(Y) \sum_{I^{\prime} \subseteq I, J^{\prime} \subseteq J} a_{I^{\prime} J^{\prime}}^{K} \\
& =\sum_{I, J \subseteq[n-1]} A_{I, J}^{K} M_{I}(X) M_{J}(Y) .
\end{aligned}
$$

As a result, $A_{I, J}^{\emptyset}$ is the coefficient in $M_{I}(X) M_{J}(Y)$ of $F_{\emptyset}(X Y)$ which we know is equal to $\sum_{\lambda \vdash n} s_{\lambda}(X) s_{\lambda}(Y)$ (see above). Finally use Equation (5) to get

$$
A_{I, J}^{\emptyset}=\sum_{\lambda \vdash n} K_{\lambda \operatorname{comp}(I)} K_{\lambda \operatorname{comp}(J)} .
$$

It remains to prove that $A_{I, J}^{\emptyset}=m_{\operatorname{comp}(I), \operatorname{comp}(J)}$. The combinatorial interpretation of [11] states that $b_{I J}^{K}$ is the number of non-negative integer matrices $M$ with row and column sums equal to $\operatorname{comp}(I)$ and $\operatorname{comp}(J)$ respectively and with the word obtained by reading the entries of $M$ row by row from top to bottom equal to $K$ (zero entries being omitted). But according to Equation (1) $A_{I, J}^{\emptyset}=\sum_{K \subseteq[n-1]} b_{I J}^{K}$. We get Equation (12).

In order to prove Equation (13), it suffices to notice that the interpretation of [11] generalises well to three dimensional arrays.

A bijective proof of Equation (13) is provided in [2]. The proof involves semistandard Young tableaux and Littlewood-Richardson tableaux. Our approach allows us to recover these results immediately.

\section{Descent algebra of the hyperoctahedral group}

\subsection{Signed permutations and domino tableaux}

Let $B_{n}$ be the hyperoctahedral group of order $n . B_{n}$ is composed of all permutations $\pi$ on the set $\{-n, \cdots,-2,-1,0,1,2, \cdots, n\}$ such that for all $i$ in $\{0\} \cup[n], \pi(-i)=-\pi(i)$ (in particular $\pi(0)=0$ ). As a result, such permutations usually referred to as signed permutations are entirely described by their 
restriction to $[n]$. The descent set of $\pi$ is the subset of $\{0\} \cup[n-1]$ defined by $\operatorname{Des}(\pi)=\{0 \leq i \leq n-1 \mid \pi(i)>\pi(i+1)\}$. The main difference with respect to the case of the symmetric group is the possible descent in position 0 when $\pi(1)$ is a negative integer. We denote $D_{I}^{B}$ (resp. $B_{I}^{B}$ ) the formal sum in $\mathbb{C} B_{n}$ of the signed permutations $\pi$ with $\operatorname{Des}(\pi)=I$ (resp. $\operatorname{Des}(\pi) \subseteq I)$. Let $c_{I_{1}, I_{2}, \cdots, I_{p}}^{K}=$ $\left[D_{K}^{B}\right] \prod_{j=1}^{p} D_{I_{j}}^{B}$ (resp. $e_{I_{1}, I_{2}, \cdots, I_{p}}^{K}=\left[B_{K}^{B}\right] \prod_{j=1}^{p} B_{I_{j}}^{B}$ ) be the structure constants of Solomon's descent algebra of type B for $I_{1}, \cdots, I_{p}, K \subseteq\{0\} \cup[n-1]$.

Remark 3.1. These constants verify

$$
\sum_{I^{\prime} \subseteq I, J^{\prime} \subseteq J} c_{I^{\prime} J^{\prime}}^{K}=\sum_{K^{\prime} \supseteq K} e_{I J}^{K^{\prime}}
$$

For $\lambda \vdash 2 n$, a standard domino tableau $T$ of shape $\lambda$ is a Young diagram of shape $\lambda$ tiled by dominoes, i.e. $2 \times 1$ or $1 \times 2$ rectangles filled with the elements of $[n]$ such that the entries are strictly increasing along the rows and down the columns. Denote $S D T(\lambda)$ the set of standard domino tableaux of shape $\lambda$. We denote $\mathcal{P}^{0}(n)$ the set of partitions $\lambda \vdash 2 n$ whose associated Young diagram may be fully tiled by dominoes. Such partitions are usually called empty 2-core partitions. A standard domino tableau $T$ has a descent in position $i>0$ if $i+1$ lies strictly below $i$ in $T$ and has descent in position 0 if the domino filled with 1 is vertical. We denote $\operatorname{Des}(T)$ the set of all its descents. For $\lambda$ in $\mathcal{P}^{0}(n)$ and $I \subseteq\{0\} \cup[n-1]$, denote $d_{\lambda I}^{B}$ the number of standard domino tableaux of shape $\lambda$ and descent set $I$.

Example 3.2. The following standard domino tableaux have shape $(5,5,4,1,1)$ and descent set $\{0,3,5,6\}$.
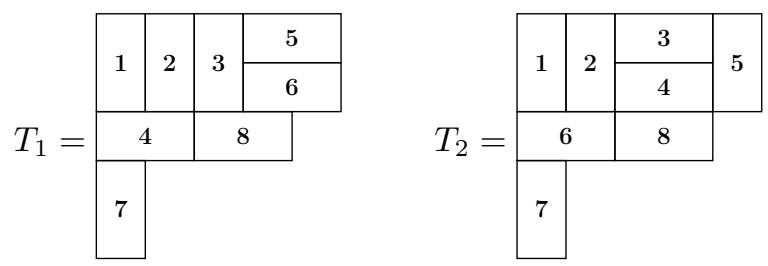

There is a natural analogue of the RSK-correspondence for signed permutations involving domino tableaux. Barbash and Vogan ([3]) built a bijection between signed permutations of $B_{n}$ and pairs of standard domino tableaux of equal shape in $\mathcal{P}^{0}(n)$. An independent development on the subject is due to Garfinkle in $[8,9,10]$. Van Leeuwen shows in [23] that the two approaches are actually equivalent. See also Stanton and White in [21] for a more general treatment of rim hook tableaux. Taşkin ([22, Prop. 26]) showed that the two standard domino tableaux associated to a signed permutation $\pi$ by the algorithm of Barbash and Vogan have respective descent sets Des $\left(\pi^{-1}\right)$ and Des $(\pi)$. As a result of this descent preserving property, we have the following analogue to Equation (2).

$$
c_{I J}^{\emptyset}=\sum_{\lambda \in \mathcal{P}^{0}(n)} d_{\lambda I}^{B} d_{\lambda J}^{B}
$$




\subsection{Quasisymmetric functions of type $B$ and main results}

Chow defines in [7] an analogue of Gessel's algebra of quasisymmetric functions that is dual to the Solomon's descent algebra of type B.

Let $X=\left\{\cdots, x_{-i}, \cdots, x_{-1}, x_{0}, x_{1}, \cdots, x_{i}, \cdots\right\}$ be an alphabet of commutative indeterminates with $x_{-i}=x_{i}$ and $I$ be a subset of $\{0\} \cup[n-1]$, he defines:

$$
M_{I}^{B}(X)=\sum_{\substack{0 \leq i_{1} \leq i_{2} \leq \ldots \leq i_{n} \\ j \in I \Leftrightarrow i_{j}<i_{j+1}}} x_{i_{1}} x_{i_{2}} \ldots x_{i_{n}}, \quad F_{I}^{B}(X)=\sum_{\substack{0 \leq i_{1} \leq i_{2} \leq \ldots \leq i_{n} \\ j \in I \Rightarrow i_{j}<i_{j+1}}} x_{i_{1}} x_{i_{2}} \ldots x_{i_{n}} .
$$

where $i_{0}=0$. Note the particular role played by variable $x_{0}$.

Example 3.3. Let $n=2$ and $X=\left\{x_{-2}, x_{-1}, x_{0}, x_{1}, x_{2}\right\}$ then $F_{\emptyset}=x_{0}^{2}+x_{1}^{2}+$ $x_{2}^{2}+x_{0} x_{1}+x_{0} x_{2}+x_{1} x_{2}, F_{\{1\}}=x_{0} x_{1}+x_{0} x_{2}+x_{1} x_{2}, F_{\{0\}}=x_{1}^{2}+x_{2}^{2}+x_{1} x_{2}$ and $F_{\{0,1\}}=x_{1} x_{2}$.

Remark 3.4. Prior to Chow, Poirier ([18]) introduced an alternative type $B$ analogue of the quasisymmetric functions used for example in [1]. However Poirier's quasisymmetric functions are dual to the Mantaci-Reutenauer algebra and not Solomon's descent algebra of type B. See [17] for further details. The question of finding a similar development as in the present paper for Poirier's quasisymmetric functions and the Mantaci-Reutenauer algebra remains open.

Let $Y$ be a second copy of $X$. Chow uses the theory of $P$-partitions of type B to show in [7, Thm. 2.3.4] that for $K \subseteq\{0\} \cup[n-1]$

$$
F_{K}^{B}(X Y)=\sum_{I, J \subseteq\{0\} \cup[n-1]} c_{I, J}^{K} F_{I}^{B}(X) F_{J}^{B}(Y) .
$$

Theorem 3.5. Equation (16) is a consequence of Equation (17).

The irreducible characters of $B_{n}$ are naturally indexed by partitions of $\mathcal{P}^{0}(n)$ (see e.g. [15, I, Appendix B]). Denote $\psi^{\lambda}$ the character indexed by $\lambda \in \mathcal{P}^{0}(n)$ and define the Kronecker coefficients of $\mathbf{B}_{\mathbf{n}}, g^{B}(\lambda, \mu, \nu)=$ $1 /\left|B_{n}\right| \sum_{\omega \in B_{n}} \psi^{\lambda}(\omega) \psi^{\mu}(\omega) \psi^{\nu}(\omega)$. A more general form of Theorem 3.5 can be stated as follows.

Theorem 3.6. Let $I, J$ and $K \subseteq\{0\} \cup[n-1]$. The following formula holds

$$
c_{I, J, K}^{\emptyset}=\sum_{\lambda, \mu, \nu \in \mathcal{P}^{0}(n)} g^{B}(\lambda, \mu, \nu) d_{\lambda I}^{B} d_{\mu J}^{B} d_{\nu K}^{B}
$$

The proof of Theorems 3.5 and 3.6 uses Equation (17) and generating functions for domino tableaux. We detail it in the two following sections.

\subsection{Domino functions}

\subsubsection{Definition}

Generating functions for domino tableaux sometimes called domino functions are well studied objects (see e.g. [13]). We introduce a modified definition 
to get an analogue of Schur functions verifying Equations (20) and (22) that we need to prove Theorem 3.6. Our development differs by the addition of " 0 " entries to the domino tableaux in some cases.

A semistandard domino tableau $\mathrm{T}$ of shape $\lambda \in \mathcal{P}^{0}(n)$ and weight $w(T)=\mu=\left(\mu_{0}, \mu_{1}, \mu_{2}, \cdots\right)$ with $\mu_{i} \geq 0$ and $\sum_{i} \mu_{i}=n$ is a tiling of the Young diagram of shape $\lambda$ with horizontal and vertical dominoes labelled with integers in $\{0,1,2, \cdots\}$ such that labels are non decreasing along the rows, strictly increasing down the columns and exactly $\mu_{i}$ dominoes are labelled with $i$. If the top leftmost domino is vertical, it cannot be labelled 0 (we leave it to the reader to check that the only possible sub-pattern of dominos with label 0 in a semistandard domino tableau is a row composed of horizontal dominos). Denote $S S D T(\lambda)$ the set of semistandard domino tableaux of shape $\lambda$ and $K_{\lambda \mu}^{B}$ the number of semistandard domino tableaux of shape $\lambda$ and weight $\mu$.

The standardisation $T^{s t}$ of a semistandard domino tableau $T$ of weight $\mu$ is the standard domino tableau obtained by relabelling the dominoes of $T$ with $1,2, \cdots, n$ such that the dominoes labelled with $i_{m}=\min \left\{i \mid \mu_{i}>0\right\}$ are relabelled with $1,2, \cdots, \mu_{i_{m}}$ from left to right and so on.

Example 3.7. Figure 5 shows a semistandard domino tableau of weight $\mu=$ $(2,0,2,0,0,4,0,1)$ and its standardisation.

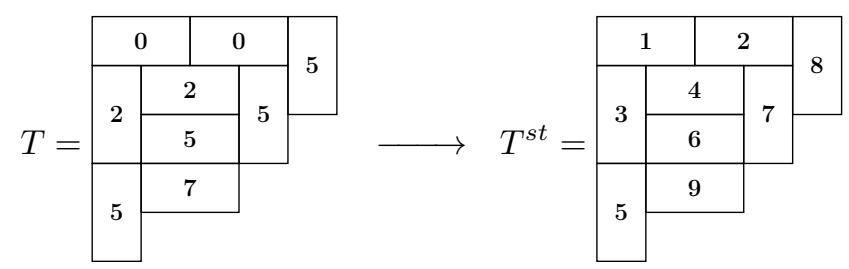

Figure 1: A semistandard domino tableau and its standardisation.

Definition 3.8. Given alphabet $X$ and a semistandard domino tableau $T$ of weight $\mu$, denote $X^{T}$ the monomial $x_{0}^{\mu_{0}} x_{1}^{\mu_{1}} x_{2}^{\mu_{2}} \ldots$. For $\lambda \in \mathcal{P}^{0}(n)$ we call the domino function indexed by $\lambda$ the function defined in the alphabet $X$ by

$$
\mathcal{G}_{\lambda}(X)=\sum_{T \in S S D T(\lambda)} X^{T}
$$

\subsubsection{Expansion into Chow's fundamental and monomial bases}

We have the following proposition.

Proposition 3.9. Domino functions admit the following expansion into Chow's fundamental and monomial bases.

$$
\mathcal{G}_{\lambda}=\sum_{I \subseteq\{0\} \cup[n-1]} d_{\lambda I}^{B} F_{I}^{B}=\sum_{I \subseteq\{0\} \cup[n-1]} K_{\lambda \operatorname{comp}(I)}^{B} M_{I}^{B} .
$$


In order to prove Proposition 3.9 we need the following lemma.

Lemma 3.10. Let $T_{0}$ be a standard domino tableau. Then the fundamental quasisymmetric function $F_{\text {Des }\left(T_{0}\right)}^{B}$ is the generating function for semistandard domino tableaux $T$ such that $T^{\text {st }}=T_{0}$, i.e.

$$
F_{D e s\left(T_{0}\right)}^{B}=\sum_{T^{s t}=T_{0}} X^{T}
$$

Proof. Given a fixed standard domino tableau $T_{0}$, we reverse the standardisation operation to obtain the set of semistandard domino tableaux with standardisation $T_{0}$. Since the standardisation operation is shape and domino tiling preserving, any such semistandard tableau $T$ may be identified by the sequence of its entries $i_{1}, i_{2}, \ldots i_{n}$.

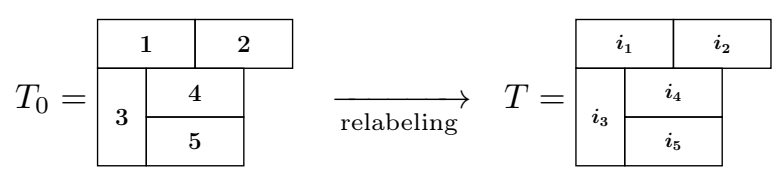

Figure 2: A standard domino tableau $T_{0}$ and the template for its semistandard preimages.

Relabel the entries of $T_{0}$ with the sequence $i_{1}, i_{2}, \ldots, i_{n}$ such that $k$ is mapped to $i_{k}$, as depicted on Figure 2. According to the standardisation procedure, we have $0 \leq i_{1} \leq i_{2} \leq \ldots \leq i_{n}$ and $0<i_{1}$ if the top leftmost domino is vertical. Furthermore such a sequence needs to verify additional conditions to give a valid semistandard domino tableau with standardisation equal to $T_{0}$. Consider each constraint $i_{k} \leq i_{k+1}$ separately. If $i_{k}<i_{k+1}$ then locally the sequence gives a valid semistandard domino tableau (entries are increasing along the row and down the columns) and the standardisation process provides the expected result.

We look at the case $i_{k}=i_{k+1}$ and show two properties.

- First, if $i_{k}=i_{k+1}$ then $k \notin \operatorname{Des}\left(T_{0}\right)$. Indeed assume $k \in \operatorname{Des}\left(T_{0}\right)$. Domino $k$ has to lay in a higher row than $k+1$ as in Figure 3.

But domino $i_{k+1}$ may not be outside the green area defined in Figure 3 as the entries in the tableau are strictly increasing down the columns. As a consequence, domino $i_{k+1}$ has to be on the left of and below domino $i_{k}$, as in Figure 3, picture (c). This is a contradiction since according to the definition of the standardisation operation, we should relabel the domino $i_{k+1}$ before the domino $i_{k}$ with such a pattern and $T_{0}$ is not the standardisation of such a tableau. As a result $k \in \operatorname{Des}\left(T_{0}\right)$ implies $i_{k}<i_{k+1}$.

- Secondly, when $k \notin \operatorname{Des}\left(T_{0}\right)$ any sequence with $i_{k}=i_{k+1}$ locally gives a valid semistandard tableau whose standardisation is equal to $T_{0}$. Indeed, 

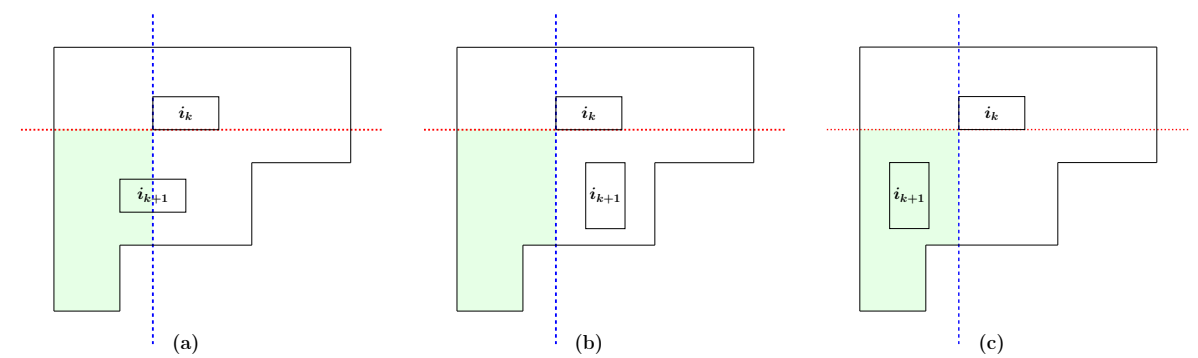

Figure 3: Layouts with a descent in position $k$. Green area corresponds to positions of domino $i_{k+1}$ which do not violate the property of increasing entries along the rows and down the columns when $i_{k}=i_{i+1}$. In pictures (a) and (b) domino $i_{k+1}$ lays outside the green area. Picture (c) shows a valid layout of dominoes $i_{k}$ and $i_{k+1}$.

if $k \notin \operatorname{Des}\left(T_{0}\right)$, domino $k$ does not lay in a higher row than $k+1$.

As a result, domino $i_{k+1}$ cannot be outside the green area defined in Figure 4 as entries are increasing along the rows and down the columns, i.e. $i_{k+1}$ is to the right of and above domino $i_{k}$. This makes sure that $i_{k}$ is relabelled before $i_{k+1}$ in the standardisation procedure.

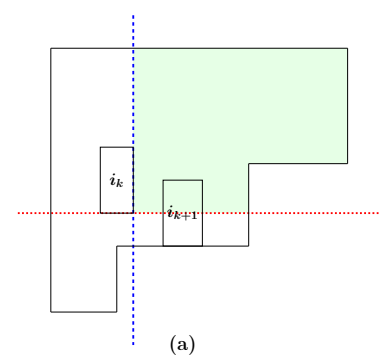

(a)

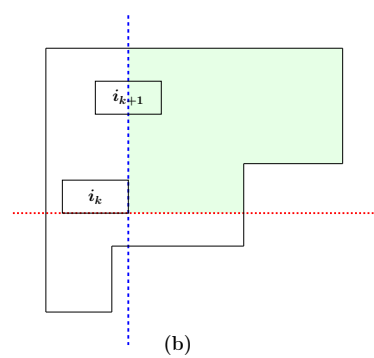

(b)

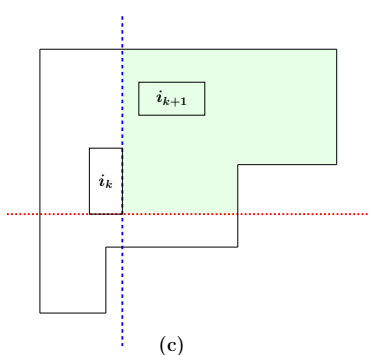

(c)

Figure 4: Layouts with no descent in position $k$. Green area corresponds to positions of domino $i_{k+1}$ which do not violate the property of increasing entries along the rows and down the columns when $i_{k}=i_{i+1}$. In pictures (a) and (b) domino $i_{k+1}$ lays outside the green area. Picture (c) shows a valid layout of dominoes $i_{k}$ and $i_{k+1}$.

As a consequence the preimages of $T_{0}$ can be identified as the sequences $0 \leq i_{1} \leq i_{2} \leq \ldots \leq i_{n}$ such that $k \in \operatorname{Des}\left(T_{0}\right) \Rightarrow i_{k}<i_{k+1}$ for $k \geq 0$ and $i_{0}=0$. Consider the constraints all together to ensure that such a sequence globally gives a valid semistandard tableau whose standardisation is equal to $T_{0}$.

We are now ready to prove Proposition 3.9.

Proof of Proposition 3.9. Classify the set of semistandard domino tableaux according to their standardisation and add the monomials corresponding to the 
tableaux in the same class. Using Lemma 3.10 we obtain the first part of the Proposition.

$$
\begin{aligned}
\mathcal{G}_{\lambda}(X) & =\sum_{T \in S S D T(\lambda)} X^{T} \\
& =\sum_{T_{0} \in S D T(\lambda)} \sum_{T \in S S D T(\lambda), T^{s t}=T_{0}} X^{T} \\
& \stackrel{(21)}{=} \sum_{T_{0} \in S D T(\lambda)} F_{D e s\left(T_{0}\right)}^{B}(X) \\
& =\sum_{I \subseteq\{0\} \cup[n-1]} d_{\lambda I}^{B} F_{I}^{B}(X) .
\end{aligned}
$$

To get the second part of the proposition, relabel the entries of a semistandard tableau $T$ with successive integers $0,1,2, \ldots$ such that inequalities and equalities between entries in $T$ are preserved after relabelling. If the top leftmost domino is vertical skip 0 . This operation removes the zeros in the weight of the tableau except, possibly, the first one. Denote $\widetilde{T}$ the resulting tableau and for $\lambda \in \mathcal{P}^{0}(n), \widetilde{S S D T}(\lambda)$ the set of semistandard domino tableaux of shape $\lambda$ such that $\widetilde{T}=T$. The second part of the statement is a consequence of the fact that the monomial quasisymmetric function $M_{\operatorname{set}\left(w\left(T_{0}\right)\right)}^{B}$ is precisely the generating function for all semistandard domino tableaux $T$ mapped to the same $T_{0} \in \widetilde{S S D T}(\lambda)$ by this operation.

$$
\begin{aligned}
& \mathcal{G}_{\lambda}(X)=\sum_{T \in S S D T(\lambda)} X^{T} \\
& =\sum_{T_{0} \in \widehat{S S D T}(\lambda)} \sum_{T \in S S D T(\lambda), \widetilde{T}=T_{0}} X^{T} \\
& =\sum_{T_{0} \in \widehat{S S D T}(\lambda)} M_{\text {set }\left(w\left(T_{0}\right)\right)}^{B} \\
& =\sum_{I \subseteq\{0\} \cup[n-1]} K_{\lambda \operatorname{comp}(I)}^{B} M_{I}^{B}(X) .
\end{aligned}
$$

Equation (20) is fully proven.

\subsubsection{Cauchy identities}

There is a well known (not descent preserving) bijection between semistandard domino tableaux of weight $\mu$ and bi-tableaux, i.e. pairs of semistandard Young tableaux of respective weights $\mu^{-}$and $\mu^{+}$such that $\mu_{i}^{+}+\mu_{i}^{-}=\mu_{i}$ for all $i$ (see e.g. [5, Algorithm 6.1]). The respective shapes of the two Young tableaux depend only on the shape of the initial semistandard domino tableau. Denote $\left(T^{-}, T^{+}\right)$the bi-tableau associated to a semistandard domino tableau 
$T$ of shape $\lambda$ and $\left(\lambda^{-}, \lambda^{+}\right)$their respective shapes. $\left(T^{-}, T^{+}\right)$(resp. $\left.\left(\lambda^{-}, \lambda^{+}\right)\right)$ is the 2-quotient of $T$ (resp. $\lambda$ ). Semistandard Young tableaux $T^{-}$and $T^{+}$ are built by filling each box of $T$ (a domino is composed of two boxes) by a '-, or a ' + ' sign such that the top leftmost box is filled with '-' and two adjacent boxes have opposite signs. $T^{-}$(resp. $T^{+}$) is obtained from the sub tableau of $T$ composed of the dominoes with top rightmost box filled with '-' (resp. '+').

Remark 3.11. If the top leftmost domino of a semistandard domino tableau $T$ is vertical (resp. horizontal), its label is used for $T^{-}$(resp. $\left.T^{+}\right)$. Therefore not labelling the top leftmost domino with 0 if it is vertical is sufficient to ensure that only $T^{+}$may have entries equal to 0 and any bi-tableau with $T^{+}$containing entries equal to 0 gives a valid semistandard domino tableau according to our constraints. As a result, in our setup, the bijection is actually between semistandard domino tableaux indexed by labels in $\{0,1,2, \cdots\}$ and pairs of semistandard Young tableaux indexed respectively by $\{1,2, \cdots\}$ and $\{0,1,2, \cdots\}$.

Example 3.12. Figure 5 shows a semistandard domino tableau of weight $\mu=$ $(2,0,2,0,0,4,0,1)$ and its 2-quotient.

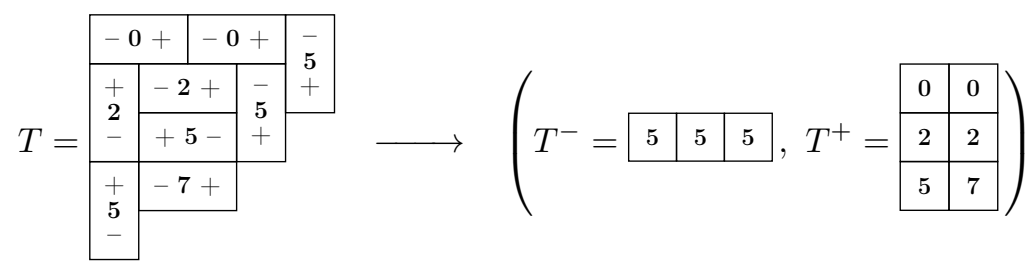

Figure 5: A semistandard domino tableau and its 2-quotient.

Denote $X^{-}=\left\{x_{-i}\right\}_{i>0}$ and $X^{+}=\left\{x_{i}\right\}_{i \geq 0}$ (note that $X^{-}=X^{+} \backslash\left\{x_{0}\right\}$ as $\left.x_{-i}=x_{i}\right)$.

Proposition 3.13. For $\lambda \in \mathcal{P}^{0}(n)$, the domino function $\mathcal{G}_{\lambda}$ and the Schur symmetric functions are related through

$$
\mathcal{G}_{\lambda}(X)=s_{\lambda^{-}}\left(X^{-}\right) s_{\lambda^{+}}\left(X^{+}\right) .
$$

Proof. According to the definition of the 2-quotient above and Remark 3.11, one can get

$$
\mathcal{G}_{\lambda}(X)=\sum_{\substack{\operatorname{shape}\left(T^{-}\right)=\lambda^{-} \\ \text {shape }\left(T^{+}\right)=\lambda^{+}}} X^{-T^{-}} X^{+^{+}}
$$

where the sum is on all pairs of semistandard Young tableau $T^{-}$and $T^{+}$of shape $\lambda^{-}$and $\lambda^{+}$such that no entry of $T^{-}$is equal to 0 . 
Finally we provide analogues of the Cauchy identities for domino functions.

Proposition 3.14. The domino functions $\mathcal{G}_{\lambda}(X Y)$ verify the following identities

$$
\begin{aligned}
\mathcal{G}_{(2 n)}(X Y) & =\sum_{\lambda \in \mathcal{P}^{0}(n)} \mathcal{G}_{\lambda}(X) \mathcal{G}_{\lambda}(Y), \\
\mathcal{G}_{\lambda}(X Y) & =\sum_{\mu, \nu \in \mathcal{P}^{0}(n)} g^{B}(\lambda, \mu, \nu) \mathcal{G}_{\mu}(X) \mathcal{G}_{\nu}(Y) .
\end{aligned}
$$

Proof. Note that

$$
(X Y)^{+}=\left\{x_{i} y_{j}\right\}_{(i, j) \geq(0,0)}=\left\{x_{0} y_{j}\right\}_{j \geq 0} \cup\left\{x_{i} y_{j}\right\}_{i>0, j \in \mathbb{Z}}=X^{+} Y^{+} \cup X^{-} Y^{-} .
$$

Using Proposition 3.13 we get the first identity.

$$
\begin{aligned}
\mathcal{G}_{(2 n)}(X Y) & =s_{(n)}\left((X Y)^{+}\right) \\
& =s_{(n)}\left(X^{-} Y^{-} \cup X^{+} Y^{+}\right) \\
& =\sum_{k=0}^{n} s_{(k)}\left(X^{-} Y^{-}\right) s_{(n-k)}\left(X^{+} Y^{+}\right) \\
& =\sum_{k=0}^{n} \sum_{\lambda^{-} \vdash k} s_{\lambda^{-}}\left(X^{-}\right) s_{\lambda^{-}}\left(Y^{-}\right) \sum_{\lambda+\vdash n-k} s_{\lambda^{+}}\left(X^{+}\right) s_{\lambda^{+}}\left(Y^{+}\right) \\
& =\sum_{\lambda \in \mathcal{P}^{0}(n)} s_{\lambda^{-}}\left(X^{-}\right) s_{\lambda^{+}}\left(X^{+}\right) s_{\lambda^{-}}\left(Y^{-}\right) s_{\lambda^{+}}\left(Y^{+}\right) \\
& =\sum_{\lambda \in \mathcal{P}^{0}(n)} \mathcal{G}_{\lambda}(X) \mathcal{G}_{\lambda}(Y) .
\end{aligned}
$$

Using Proposition 3.13 and the theory of symmetric functions on wreath products we prove the second identity. Define as in [1] for any set of indeterminates $U$ and $V$ and any partition $\lambda$ of $n$ :

$$
p_{\lambda}^{+}(U, V)=\prod_{i}\left[p_{\lambda_{i}}(U)+p_{\lambda_{i}}(V)\right], \quad p_{\lambda}^{-}(U, V)=\prod_{i}\left[p_{\lambda_{i}}(U)-p_{\lambda_{i}}(V)\right] .
$$

Note that if $U \cap V=\emptyset, p_{\lambda}^{+}(U, V)=p_{\lambda}(U \cup V)$ and if $V \subseteq U, p_{\lambda}^{-}(U, V)=$ $p_{\lambda}(U \backslash V)$. Then for partitions $\lambda \in \mathcal{P}^{0}(n)$ and $\mu \in \mathcal{P}^{0}(n)$ of 2 -quotient $\left(\mu^{-}, \mu^{+}\right)$, denote $\psi_{\mu}^{\lambda}$ the common value of character $\psi^{\lambda}$ on all signed permutations of cycle type $\left(\mu^{-}, \mu^{+}\right)$. According to [15, I, Appendix B],

$$
p_{\mu^{-}}^{-}(U, V) p_{\mu^{+}}^{+}(U, V)=\sum_{\lambda \in \mathcal{P}^{0}(n)} \psi_{\mu}^{\lambda} s_{\lambda^{-}}(V) s_{\lambda^{+}}(U) .
$$

Set $U=X^{+}$and $V=X^{-}$and use Proposition 3.13 to get for any $\mu \in \mathcal{P}^{0}(n)$

$$
p_{\mu^{+}}(X)\left(x_{0}\right)^{n-\left|\mu^{+}\right|}=\sum_{\lambda \in \mathcal{P}^{0}(n)} \psi_{\mu}^{\lambda} \mathcal{G}_{\lambda}(X) .
$$


But $p_{\mu^{+}}(X Y)\left(x_{0} y_{0}\right)^{n-\left|\mu^{+}\right|}=p_{\mu^{+}}(X)\left(x_{0}\right)^{n-\left|\mu^{+}\right|} p_{\mu^{+}}(Y)\left(y_{0}\right)^{n-\left|\mu^{+}\right|}$. We get

$$
\sum_{\lambda \in \mathcal{P}^{0}(n)} \psi^{\lambda} \mathcal{G}_{\lambda}(X Y)=\sum_{\mu, \nu \in \mathcal{P}^{0}(n)} \psi^{\mu} \psi^{\nu} \mathcal{G}_{\mu}(X) \mathcal{G}_{\nu}(Y) .
$$

That yields the second identity.

\subsection{Proof of Theorems 3.5 and 3.6}

Both Theorem 3.5 and Theorem 3.6 are consequences of Equation (17) and Proposition 3.14.

Proof of Theorem 3.5. Decompose the domino functions $\mathcal{G}_{(2 n)}(X Y)$ in two ways.

$$
\begin{aligned}
& \mathcal{G}_{(2 n)}(X Y)=F_{\emptyset}^{B}(X Y)=\sum_{I, J} c_{I, J}^{\emptyset} F_{I}(X) F_{J}(Y) \\
& \mathcal{G}_{(2 n)}(X Y)=\sum_{\lambda \in \mathcal{P}^{0}(n)} \mathcal{G}_{\lambda}(X) \mathcal{G}_{\lambda}(Y)=\sum_{I, J} \sum_{\lambda \vdash n} d_{\lambda I}^{B} d_{\lambda J}^{B} F_{I}^{B}(X) F_{J}^{B}(Y) .
\end{aligned}
$$

Proof of Theorem 3.6. Similarly,

$$
\begin{aligned}
\mathcal{G}_{(2 n)}(X Y Z) & =F_{\emptyset}^{B}(X Y Z)=\sum_{I, J, K} c_{I, J, K}^{\emptyset} F_{I}^{B}(X) F_{J}^{B}(Y) F_{K}^{B}(Z) \\
\mathcal{G}_{(2 n)}(X Y Z) & =\sum_{\nu \in \mathcal{P}^{0}(n)} \mathcal{G}_{\nu}(X Y) \mathcal{G}_{\nu}(Z) \\
& =\sum_{\lambda, \mu, \nu \in \mathcal{P}^{0}(n)} g^{B}(\lambda, \mu, \nu) \mathcal{G}_{\lambda}(X) \mathcal{G}_{\mu}(Y) \mathcal{G}_{\nu}(Z) \\
& =\sum_{I, J, K} \sum_{\lambda, \mu, \nu \in \mathcal{P}^{0}(n)} g^{B}(\lambda, \mu, \nu) d_{\lambda I}^{B} d_{\mu J}^{B} d_{\nu K}^{B} F_{I}^{B}(X) F_{J}^{B}(Y) F_{K}^{B}(Z) .
\end{aligned}
$$

Remark 3.15. Theorem 3.5 is a special case of Theorem 3.6. However, we felt that an independent proof that does not use Kronecker coefficients but only the elementary first identity of Proposition 3.14 was of interest.

\subsection{A corollary to Theorem 3.6}

For $I \subseteq\{0\} \cup[n-1]$, denote $|I|$ the number of elements in $I$. The structure constants $e_{I J}^{K}$ also count templates $M$ with non-negative integer entries of the following type.

$$
M=\left(m_{i, j}\right)=\left(\begin{array}{cccc}
a_{0,0} & a_{0,1} & \ldots & a_{0,|I|} \\
& b_{1,1} & \ldots & b_{1,|I|} \\
a_{1,0} & a_{1,1} & \ldots & a_{1,|I|} \\
\vdots & & & \vdots \\
& b_{|J|, 1} & \ldots & b_{|J|,|I|} \\
a_{|J|, 0} & a_{|J|, 1} & \ldots & a_{|J|,|I|}
\end{array}\right)
$$


Given $q_{s}=a_{s, 0}+\sum_{k}\left(a_{s, k}+b_{s, k}\right)$ and $q_{0}=a_{0,0}+\sum_{k} a_{0, k}$ we denote $q(M)=$ $\left(q_{0}, q_{1}, \ldots q_{|I|}\right)$ the row sums. The column sums $p(M)=\left(p_{0}, p_{1}, \ldots p_{|J|}\right)$ verify $p_{j}=a_{0, j}+\sum_{k}\left(a_{k, s}+b_{k, s}\right)$ and $p_{0}=a_{0,0}+\sum_{k} a_{k, 0}$. Successive reading of $a$-lines from left to right, and $b$-lines from right to left yields the reading word. Denote $n_{I, J}^{K}$ the number of such templates with row sums $I$, column sums $J$ and reading word $K$ and $n_{I, J}=\sum_{K} n_{I, J}^{K}$. Bergeron and Bergeron in [4] prove that $n_{I, J}^{K}=e_{I J}^{K}$. We get as a corollary to Theorem 3.6:

Corollary 3.16. Let $I, J$ and $K \subseteq\{0\} \cup[n-1]$. The numbers $n_{I, J}$ and $n_{I, J}^{K}$ verify

$$
\begin{aligned}
n_{I, J} & =\sum_{\lambda} K_{\lambda \operatorname{comp}(I)}^{B} K_{\lambda \operatorname{comp}(J)}^{B} \\
\sum_{R, D \subseteq\{0\} \cup[n-1]} n_{I, J}^{D} n_{D, K}^{R} & =\sum_{\lambda, \mu, \nu \vdash n} g^{B}(\lambda, \mu, \nu) K_{\lambda \operatorname{comp}(I)}^{B} K_{\mu \operatorname{comp}(J)}^{B} K_{\nu \operatorname{comp}(K)}^{B} .
\end{aligned}
$$

Proof. Considering relations (16) and (18) in the monomial basis one gets

$$
\begin{aligned}
\sum_{I, J, \quad} \sum_{I^{\prime} \subseteq I, J^{\prime} \subseteq J} c_{I^{\prime}, J^{\prime}}^{\emptyset} M_{I}^{B}(X) M_{J}^{B}(Y)= \\
\sum_{I, J} \sum_{\lambda \vdash n} K_{\lambda \operatorname{comp}(I)}^{B} K_{\lambda \operatorname{comp}(J)}^{B} M_{I}^{B}(X) M_{J}^{B}(Y),
\end{aligned}
$$

and

$$
\begin{aligned}
& \sum_{I, J, K} \sum_{I^{\prime} \subseteq I, J^{\prime} \subseteq J, K^{\prime} \subseteq K} c_{I^{\prime}, J^{\prime}, K^{\prime}}^{\emptyset} M_{I}^{B}(X) M_{J}^{B}(Y) M_{K}^{B}(Z)= \\
& \sum_{I, J, K} \sum_{\lambda, \mu, \nu \vdash n} g^{B}(\lambda, \mu, \nu) K_{\lambda \operatorname{comp}(I)}^{B} K_{\mu \operatorname{comp}(J)}^{B} K_{\nu \operatorname{comp}(K)}^{B} M_{I}^{B}(X) M_{J}^{B}(Y) M_{K}^{B}(Z) .
\end{aligned}
$$

The first equation of the statement is a consequence of the result of Bergeron and Bergeron and Equation (15).

$$
n_{I, J}=\sum_{K} e_{I J}^{K}=\sum_{I^{\prime} \subseteq I, J^{\prime} \subseteq J} c_{I^{\prime} J^{\prime}}^{\emptyset}=\sum_{\lambda \vdash n} K_{\lambda \operatorname{comp}(I)}^{B} K_{\lambda \operatorname{comp}(J)}^{B} .
$$

To get the second identity compute

$$
\begin{aligned}
\sum_{R, D} n_{I, J}^{D} n_{D, K}^{R} & =\sum_{R, D} e_{I, J}^{D} e_{D, K}^{R} \\
& =\sum_{R} e_{I, J, K}^{R} \\
& =\sum_{I^{\prime} \subseteq I, J^{\prime} \subseteq J, K^{\prime} \subseteq K} c_{I^{\prime}, J^{\prime}, K^{\prime}}^{\emptyset} \\
& =\sum_{\lambda, \mu, \nu \vdash n} g^{B}(\lambda, \mu, \nu) K_{\lambda \operatorname{comp}(I)}^{B} K_{\mu \operatorname{comp}(J)}^{B} K_{\nu \operatorname{comp}(K)}^{B} .
\end{aligned}
$$




\section{Skew shape partitions}

\subsection{Type $A$}

Let $n$ be a non-negative integer and $\lambda$ and $\mu$, two integer partitions such that the diagram of $\mu$ is included in the diagram of $\lambda$ and $|\lambda|-|\mu|=n$. A standard (resp. semistandard) skew Young tableau of shape $\lambda / \mu$ is a Young diagram of skew shape $\lambda / \mu$ whose boxes are filled with the elements of $[n]$ (resp. with positive integers) such that the entries are strictly increasing down the columns and along the rows (resp. strictly increasing down the columns and non decreasing along the rows). The descent set of a standard Young tableau is defined similarly as in the case of classical shapes. The skew Schur function $s_{\lambda / \mu}$ is the generating function for semistandard skew Young tableaux of shape $\lambda / \mu$

$$
s_{\lambda / \mu}(X)=\sum_{\operatorname{shape}(T)=\lambda / \mu} X^{T},
$$

where the sum is on all semistandard skew Young tableaux of shape $\lambda / \mu$. Given a skew shape $\lambda / \mu$ denote $k_{\mu \nu}^{\lambda}$ the Littlewood-Richardson coefficients i.e. the structure constants of the algebra of symmetric functions in the Schur basis. They also appear in the decomposition of skew Schur functions in terms of non-skew Schur functions

$$
s_{\lambda} s_{\mu}=\sum_{\nu} k_{\lambda \mu}^{\nu} s_{\nu}, \quad s_{\lambda / \mu}=\sum_{\nu} k_{\mu \nu}^{\lambda} s_{\nu} .
$$

Let $I$ and $J$ be two subsets of $[n-1]$. Gessel focuses in [12, Thm. 14] on the number $a_{I J}^{\lambda / \mu}$ of couples of permutations $\alpha, \beta$ such that $\alpha$ has descent set $I$, $\beta$ has descent set $J$ and $\alpha \beta$ is compatible with the skew shape $\lambda / \mu$ (i.e. $\alpha \beta$ is a correct reading word for the skew shape $\lambda / \mu$ ). We need some more notions introduced in [12] to define this properly.

Given any partial order $P$ on $[n]$, denote $L(P)$ the set of permutations $\pi \in$ $S_{n}$, such that the corresponding linear order $\pi(1)<\pi(2)<\cdots<\pi(n)$ is a linearisation of $P$. To obtain a partial order $P_{\lambda / \mu}$ from the skew shape $|\lambda / \mu|=n$ we fill the corresponding skew Young diagram by integers $[n]$ from bottom to top and from left to right and then consider relations $i>k$ if $k$ lay directly below or to the left of $i$. These relations generate the corresponding partial order $P_{\lambda / \mu}$.

Example 4.1. Let $\lambda / \mu=(4,4,3) /(2,1)$. Figure 6 shows the corresponding partial order. Firstly fill the corresponding skew Young diagram by integers [7] from bottom to top and from left to right and then rotate it by 135 degrees counterclockwise and take its vertical mirror image to get the plane presentation of the ordering $P_{(4,4,3) /(2,1)}$.

Due to Gessel [12, p. 295], $L\left(P_{\lambda / \mu}\right)$ may be identified with Young tableaux of shape $\lambda / \mu$. Given $\pi \in L\left(P_{\lambda / \mu}\right)$ construct the corresponding skew Young tableau by filling the skew shape $\lambda / \mu$ with the letters of the word $\pi^{-1}$ from bottom to top and from left to right, as in Example 4.2. The permutations from $L\left(P_{\lambda / \mu}\right)$ are called compatible with the skew shape $\lambda / \mu$. 


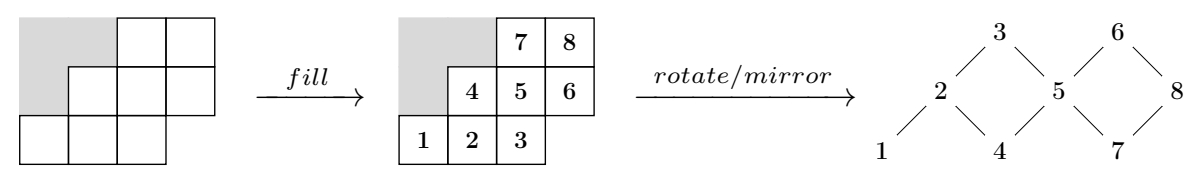

Figure 6: A partial order $P_{(4,4,3) /(2,1)}$ obtained from the skew shape $(4,4,3) /(2,1)$.

\section{Example 4.2.}

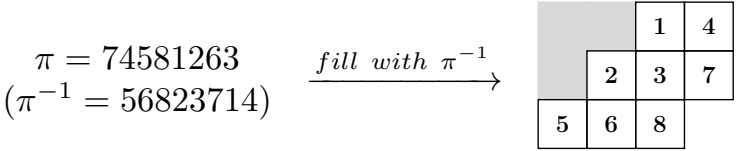

Using the bijection above, one can notice that

$$
a_{I J}^{\lambda / \mu}=\sum_{K \subseteq[|\lambda|-|\mu|-1]} d_{\lambda / \mu K} a_{I J}^{K}
$$

where $d_{\lambda / \mu K}$ is the number of standard Young tableaux of skew shape $\lambda / \mu$ and descent set $K$.

Proposition 4.3. Let $\lambda$ and $\mu$ two integer partitions such that $\lambda / \mu$ is a skew shape and $I, J \subseteq[|\lambda|-|\mu|-1]$. The coefficients $a_{I J}^{\lambda / \mu}$ verify the following equality involving the Littlewood-Richardson coefficients, the Kronecker coefficients of the symmetric group and the numbers of standard Young tableaux of given shape and descent

$$
a_{I J}^{\lambda / \mu}=\sum_{\nu, \rho, \varepsilon} k_{\mu \nu}^{\lambda} g(\nu, \rho, \varepsilon) d_{\rho I} d_{\varepsilon J}
$$

Proof. Gessel showed in [12, Thm. 14] that

$$
s_{\lambda / \mu}(X Y)=\sum_{I, J} a_{I J}^{\lambda / \mu} F_{I}(X) F_{J}(Y) .
$$

Use Equation (23) to decompose skew Schur function $s_{\lambda / \mu}(X Y)$ using LittlewoodRichardson coefficients. Then use the generalised Cauchy identity (10) and decomposition (4) to prove the proposition.

$$
\begin{aligned}
s_{\lambda / \mu}(X Y) & =\sum_{\nu} k_{\mu \nu}^{\lambda} s_{\nu}(X Y) \\
& =\sum_{\nu, \rho, \varepsilon} k_{\mu \nu}^{\lambda} g(\nu, \rho, \varepsilon) s_{\rho}(X) s_{\varepsilon}(Y) \\
& =\sum_{I, J} \sum_{\nu, \rho, \varepsilon} k_{\mu \nu}^{\lambda} g(\nu, \rho, \varepsilon) d_{\rho I} d_{\varepsilon J} F_{I}(X) F_{J}(Y) .
\end{aligned}
$$

In the following section, we prove that there is a similar formula for the hyperoctahedral group provided some constraints on the skew-shapes. 


\subsection{Type $B$}

\subsubsection{Skew domino tableaux}

Let $\lambda$ and $\mu$ be two integer partitions such that $\lambda / \mu$ is a skew shape, $|\lambda|-|\mu|=$ $2 n$ and such that the Young diagrams of shape $\mu$ and $\lambda / \mu$ (and by extension $\lambda$ ) may be tiled by horizontal and vertical dominoes. By abuse of notation, we also write $\lambda / \mu \in \mathcal{P}^{0}(n)$ when all these conditions are fulfilled. A standard skew domino tableau is a tiling of the skew Young diagram of shape $\lambda / \mu$ with horizontal and vertical dominoes labelled with integers of $[n]$ such that labels are strictly increasing along the rows and down the columns.

The bijection described in Carre and Leclerc [5] extends to the case of skew shapes. As a result, there is a bijection between standard skew domino tableaux of shape $\lambda / \mu$ and standard skew bi-tableaux of shape $\left(\lambda^{-} / \mu^{-}, \lambda^{+} / \mu^{+}\right)$. As noticed in Section 3.3.3, $\mu^{-}$and $\mu^{+}$depends only on $\mu$. One may apply the algorithm of Carre and Leclerc with an arbitrary tiling of the deleted part of shape $\mu$. Note that the bijection is not descent preserving as in the case of non-skew shapes.

Example 4.4. Decompose the following standard skew domino tableau $T$.

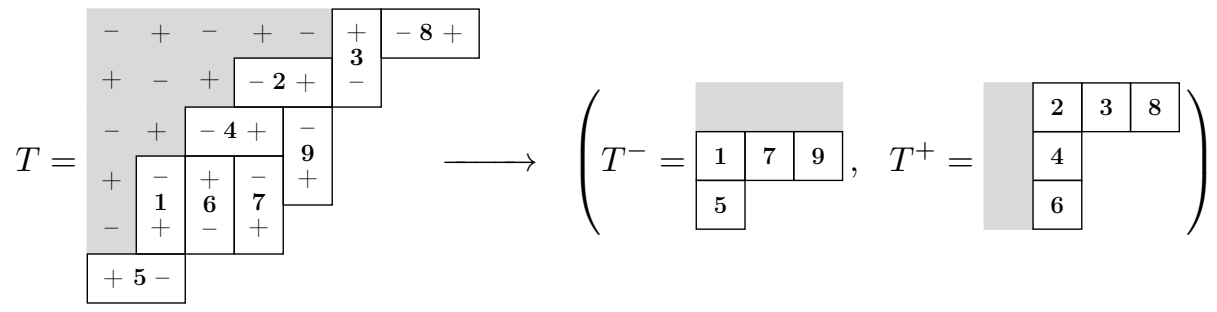

Given a standard skew domino tableau $T$, we call in the sequel negative (resp. positive) domino, a domino that goes to $T^{-}$(resp. $T^{+}$) according to the bijection above. We have the following definition.

Definition 4.5 (Descent set of a standard skew domino tableau). A standard skew domino tableau $T$ has a descent in position $i>0$ if $i+1$ lies strictly below $i$ in $T$ and has descent in position 0 if the domino filled with 1 is negative. Denote $\operatorname{Des}(T)$ the set of all the descents of $T$. For $\lambda / \mu \in \mathcal{P}^{0}(n)$ and $I \subseteq\{0\} \cup[n-1]$ denote also $d_{\lambda / \mu I}^{B}$ the number of standard skew domino tableaux of shape $\lambda / \mu$ and descent set $I$.

Example 4.6. In Example 4.4 we have Des $(T)=\{0,3,4,8\}$.

A semistandard skew domino tableau is a tiling of the skew Young diagram of shape $\lambda / \mu$ with horizontal and vertical dominoes labelled with integers in $\{0,1,2, \cdots\}$ such that labels are non decreasing along the rows, strictly increasing down the columns, and negative dominoes cannot be labelled 0 . The bijection of Carre and Leclerc [5] also generalises to semistandard domino tableaux and pairs of semistandard Young tableaux such that dominoes labelled 0 may appear only in $T^{+}$. 
Example 4.7. Decompose the following semistandard skew domino tableau.

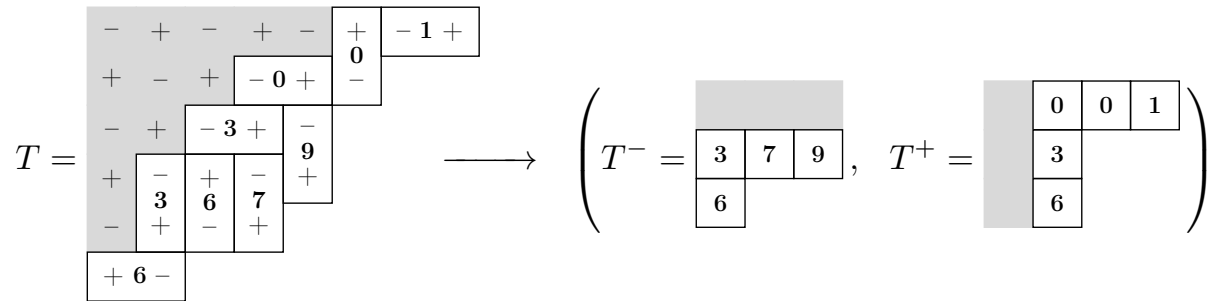

The standardisation of a semistandard skew domino tableau is defined in the same way as for non-skew shapes. We provide the following example in lieu of a formal definition.

Example 4.8. The following picture shows a semistandard skew domino tableau $T$ and its standardisation $T^{s t}$.
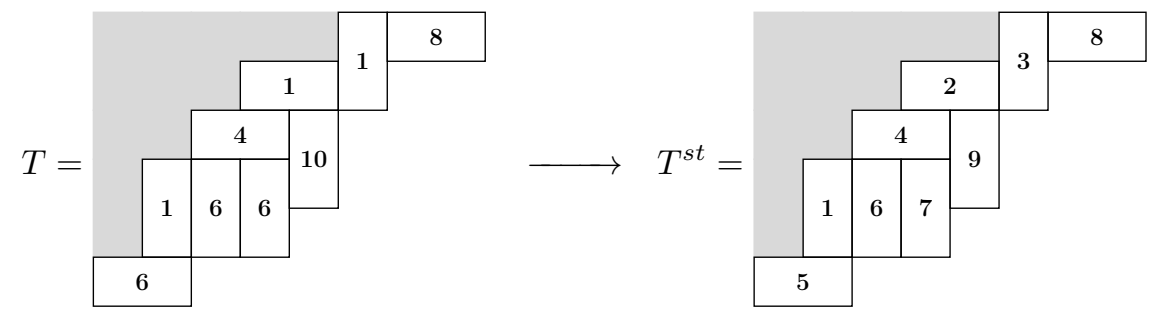

\subsubsection{Skew domino functions}

For $\lambda / \mu \in \mathcal{P}^{0}(n)$ define the skew domino function $\mathcal{G}_{\lambda / \mu}$ as the generating function for the semistandard skew domino tableaux of shape $\lambda / \mu$. Proposition 3.13 generalises well to the case of skew shapes.

Proposition 4.9. For $\lambda / \mu \in \mathcal{P}^{0}(n)$, the domino function $\mathcal{G}_{\lambda / \mu}$ and the Schur symmetric functions are related through

$$
\mathcal{G}_{\lambda / \mu}(X)=s_{\lambda^{-} / \mu^{-}}\left(X^{-}\right) s_{\lambda^{+} / \mu^{+}}\left(X^{+}\right) .
$$

Proof. This identity is a direct consequence of the bijection between semistandard skew domino tableaux of shape $\lambda / \mu$ and semistandard skew bi-tableaux of shape $\left(\lambda^{-} / \mu^{-}, \lambda^{+} / \mu^{+}\right)$and the fact that only positive dominoes may be labelled 0 .

Furthermore, we have the following proposition involving some type B analogue of the Littlewood-Richardson coefficients.

Proposition 4.10. For $\lambda / \mu \in \mathcal{P}^{0}(n), \mu \in \mathcal{P}^{0}(m)$ and $\nu \in \mathcal{P}^{0}(n)$, denote $l_{\mu \nu}^{\lambda}$ the type $\mathbf{B}$ analogues of the Littlewood-Richardson coefficients that we define as $l_{\mu \nu}^{\lambda}=k_{\mu^{-} \nu^{-}}^{\lambda^{-}} k_{\mu^{+} \nu^{+}}^{\lambda^{+}}$. Domino functions verify

$$
\mathcal{G}_{\mu} \mathcal{G}_{\nu}=\sum_{\lambda \in \mathcal{P}^{0}(n+m)} l_{\mu \nu}^{\lambda} \mathcal{G}_{\lambda}, \quad \mathcal{G}_{\lambda / \mu}=\sum_{\nu \in \mathcal{P}^{0}(n)} l_{\mu \nu}^{\lambda} \mathcal{G}_{\nu} .
$$


Proof. Use Propositions 3.13 and 4.9 to show

$$
\begin{aligned}
\mathcal{G}_{\mu}(X) \mathcal{G}_{\nu}(X) & =s_{\mu^{-}}\left(X^{-}\right) s_{\mu^{+}}\left(X^{+}\right) s_{\nu^{-}}\left(X^{-}\right) s_{\nu^{+}}\left(X^{+}\right) \\
& =\sum_{\lambda-+\left|\mu^{-}\right|+\left|\nu^{-}\right|} k_{\mu^{-} \nu^{-}}^{\lambda^{-}} s_{\lambda^{-}}\left(X^{-}\right) \sum_{\lambda \lambda^{++}\left|\mu^{+}\right|+\left|\nu^{+}\right|} k_{\mu^{+} \nu^{+}}^{\lambda^{+}} s_{\lambda^{+}}\left(X^{+}\right) \\
& =\sum_{\lambda \in \mathcal{P}^{0}(n+m)}\left(k_{\mu^{-} \nu^{-}}^{\lambda^{-}} k_{\mu^{+} \nu^{+}}^{\lambda^{+}}\right) \mathcal{G}_{\lambda}(X),
\end{aligned}
$$

and

$$
\begin{aligned}
\mathcal{G}_{\lambda / \mu}(X) & =s_{\lambda^{-} / \mu^{-}}\left(X^{-}\right) s_{\lambda^{+} / \mu^{+}}\left(X^{+}\right) \\
& =\left(\sum_{\nu^{-} \vdash\left|\lambda^{-}\right|-\left|\mu^{-}\right|} k_{\mu^{-} \nu^{-}}^{\lambda^{-}} s_{\nu^{-}}\left(X^{-}\right)\right)\left(\sum_{\nu^{+}-\left|\lambda^{+}\right|-\left|\mu^{+}\right|} k_{\mu^{+} \nu^{+}}^{\lambda^{+}} s_{\nu^{+}}\left(X^{+}\right)\right) \\
& =\sum_{\nu \in \mathcal{P}^{0}(n)}\left(k_{\mu^{-} \nu^{-}}^{\lambda^{-}} k_{\mu^{+} \nu^{+}}^{\lambda^{+}}\right) \mathcal{G}_{\nu}(X) .
\end{aligned}
$$

In order to get an analogue of Proposition 3.9, the skew shapes have to verify some additional constraints. As noticed in Remark 3.11 for non-skew shapes, the constraints of not labelling the vertical top leftmost domino of a semistandard domino tableau $T$ with 0 and the constraint that no negative domino may be labelled with 0 are equivalent. With skew shapes there is no analogue to this equivalence in the general case and we need to restrict the set of considered shapes. We proceed with the following definitions.

Definition 4.11 (Top domino). Given a (semi-)standard domino tableau T, we say that domino $d$ is a top domino of $T$ if

(i) there is no adjacent domino on the top of $d$,

(ii) there is no non-top adjacent domino to the left of $d$.

We further say that a top domino $d$ is minimum positive if $d$ is the leftmost downmost positive top domino of $T$ such that there is no adjacent (negative top) domino to the left of $d$.

Example 4.12. In Example 4.4, dominoes labelled with 1, 2, 3 and 8 are top dominoes and 2 is the minimum positive one.

We look at the following subset of $\mathcal{P}^{0}(n)$.

Definition 4.13 (Admissible skew shapes). An admissible skew shape is a skew shape $\lambda / \mu \in \mathcal{P}^{0}(n)$ that cannot be tiled with horizontal and vertical dominoes in a way that

(i) there is a minimum positive domino,

(ii) a negative top domino is placed to the right and above the minimum positive domino. 
Denote $\widetilde{\mathcal{P}^{0}}(n)$ the set of admissible skew shapes $\lambda / \mu$ such that $|\lambda|-|\mu|=n$.

Example 4.14. The shape of the standard skew domino tableau in Example 4.4 is admissible as well as the following shapes.
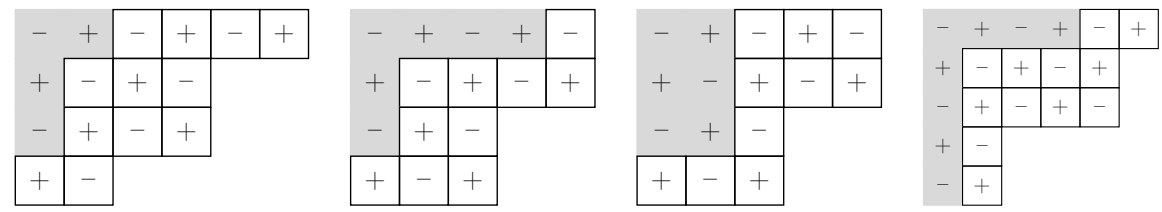

These ones are not admissible.
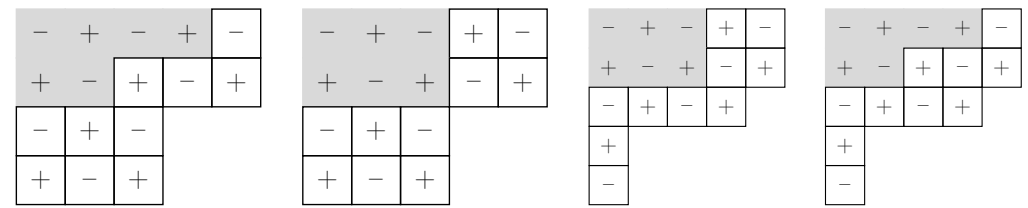

Proposition 4.15. For $\lambda / \mu \in \widetilde{\mathcal{P}^{0}}(n)$, the skew domino function $\mathcal{G}_{\lambda / \mu}$ verify

$$
\mathcal{G}_{\lambda / \mu}=\sum_{I \subseteq\{0\} \cup[n-1]} d_{\lambda / \mu I}^{B} F_{I}^{B} .
$$

Proof. The proof is adapted from the one of Proposition 3.9. The first step is to show the analogue of Lemma 3.10, i.e. that for any standard skew domino tableau $T_{0}$ of shape $\lambda / \mu$,

$$
F_{D e s\left(T_{0}\right)}^{B}=\sum_{T^{s t}=T_{0}} X^{T} .
$$

To this end one needs to show that any sequence of relabelling of the dominoes $1,2, \cdots, n$ in $T_{0}$ by $i_{1} \leq i_{2}, \leq \cdots, \leq i_{n}$ such that $k \in \operatorname{Des}\left(T_{0}\right) \Rightarrow i_{k}<i_{k+1}$ with $k \geq 0$ and $i_{0}=0$ gives a valid semistandard skew domino tableau $T$ such that $T^{s t}=T_{0}$. If the domino labelled 1 in $T_{0}$ is positive, i.e. $\min \left(\operatorname{Des}\left(T_{0}\right)\right)=k>0$, then any subsequence $0=i_{1}=i_{2}=\cdots=i_{l}(l \leq k)$ gives a valid semistandard skew domino tableau. Indeed, since $\lambda / \mu$ is admissible, none of the dominoes $2,3, \cdots, k$ are negative. As a result, no negative domino is labelled with 0 (a restriction in our definition of semistandard skew domino tableaux required to get Proposition 4.9). Other cases are similar to the proof of Lemma 3.10 and not detailed here.

Remark 4.16. The proof of Proposition 4.15 makes it clear that admissibility is required to get at the same time Proposition 4.15 and Proposition 4.9. Indeed the later requires that only positive dominoes of a semistandard skew domino tableau may be labelled with 0 . The former assumes that any sequence of dominoes labelled $1,2, \cdots, \min \left(\operatorname{Des}\left(T_{0}\right)\right)$ in a standard skew domino tableau $T$ may be relabelled with 0 to get a valid preimage of $T$ by standardisation. Only admissible shapes fulfil both conditions. 
Define for $I, J \subseteq 0 \cup[n-1]$ the coefficients $c_{I J}^{\lambda / \mu}=\sum_{K \in 0 \cup[n-1]} d_{\lambda / \mu K}^{B} c_{I J}^{K}$. Formula (24) admits the following type B analogue.

Proposition 4.17. For $\lambda / \mu \in \widetilde{\mathcal{P}^{0}}(n)$ and $I, J \subseteq 0 \cup[n-1]$, the coefficients $c_{I J}^{\lambda / \mu}$ verify

$$
\mathcal{G}_{\lambda / \mu}(X Y)=\sum_{I, J \subseteq 0 \cup[n-1]} c_{I J}^{\lambda / \mu} F_{I}^{B}(X) F_{J}^{B}(Y)
$$

Proof. Apply Proposition 4.15 and then decompose Chow's fundamental quasisymmetric function $F_{K}^{B}(X Y)$ into $F_{I}^{B}(X)$ and $F_{J}^{B}(Y)$ to prove the statement.

$$
\begin{aligned}
\mathcal{G}_{\lambda / \mu}(X Y) & =\sum_{K \subseteq\{0\} \cup[n-1]} d_{\lambda / \mu K}^{B} F_{K}^{B}(X Y) \\
& =\sum_{I, J}\left(\sum_{K} d_{\lambda / \mu K}^{B} c_{I J}^{K}\right) F_{I}^{B}(X) F_{J}^{B}(Y) \\
& =\sum_{I, J} c_{I J}^{\lambda / \mu} F_{I}^{B}(X) F_{J}^{B}(Y) .
\end{aligned}
$$

\subsubsection{A type $B$ analogue of Proposition 4.3}

We are now ready to state the main result of this section.

Theorem 4.18. For $\lambda / \mu \in \widetilde{\mathcal{P}^{0}}(n)$ and $\nu \in \mathcal{P}^{0}(n)$ denote $l_{\mu \nu}^{\lambda}$ the type $B$ analogue of the Littlewood-Richardson coefficients defined as in Proposition 4.10 by $l_{\mu \nu}^{\lambda}=$ $k_{\mu^{-} \nu^{-}}^{\lambda^{-}} k_{\mu^{+} \nu^{+}}^{\lambda^{+}}$. Then, for $I, J \subseteq 0 \cup[n-1]$, the coefficient $c_{I J}^{\lambda / \mu}$ is given by

$$
c_{I J}^{\lambda / \mu}=\sum_{\nu, \rho, \varepsilon \in \mathcal{P}^{0}(n)} l_{\mu \nu}^{\lambda} g^{B}(\nu, \rho, \varepsilon) d_{\rho I}^{B} d_{\varepsilon J}^{B} .
$$

Proof. On the one hand expand $\mathcal{G}_{\lambda / \mu}(X Y)$ using Proposition 4.17. On the other hand expand the same skew domino function using Proposition 4.10, Proposition 3.14 and Proposition 3.9. Namely

$$
\begin{aligned}
\sum_{I, J \subseteq 0 \cup[n-1]} c_{I, J}^{\lambda / \mu} F_{I}^{B}(X) F_{J}^{B}(Y) & =\mathcal{G}_{\lambda / \mu}(X Y) \\
& =\sum_{\nu \in \mathcal{P}^{0}(n)} l_{\mu \nu}^{\lambda} \mathcal{G}_{\nu}(X Y) \\
& =\sum_{\nu \in \mathcal{P}^{0}(n)} l_{\mu \nu}^{\lambda} \sum_{\rho, \varepsilon \in \mathcal{P}^{0}(n)} g^{B}(\nu, \rho, \varepsilon) \mathcal{G}_{\rho}(X) \mathcal{G}_{\varepsilon}(Y) \\
& =\sum_{\substack{I, J \subseteq 0 \cup[n-1] \\
\nu, \rho, \varepsilon \in \mathcal{P}^{0}(n)}} l_{\mu \nu}^{\lambda} g^{B}(\nu, \rho, \varepsilon) d_{\rho I}^{B} d_{\varepsilon J}^{B} F_{I}^{B}(X) F_{J}^{B}(Y)
\end{aligned}
$$




\section{Acknowledgements}

This project has been partially supported by the Vernadski scholarship.

\section{References}

[1] R. Adin, C. A. Athanasiadis, S. Elizalde, and Y. Roichman. Character formulas and descents for the hyperoctahedral group. Advances in Applied Mathematics, 87:128-169, 2017.

[2] D. Avella-Alaminosa and E. Vallejo. Kronecker products and the RSK correspondence. Discrete Mathematics, 312:1476-1486, 2012.

[3] D. Barbash and D. Vogan. Primitive ideals and orbital integrals in complex classical groups. Math. Ann., 259:153-199, 1982.

[4] F. Bergeron and N. Bergeron. A decomposition of the descent algebra of the hyperoctahedral group. Journal of Algebra, 148(1):86-97, 1992.

[5] C. Carré and B. Leclerc. Splitting the square of a Schur function into its symmetric and antisymmetric parts. Journal of Algebraic Combinatorics, 4:201-231, 1995.

[6] R. Carter. Representation theory of the 0-Hecke algebra. Journal of Algebra, 104:89-103, 1986.

[7] C. Chow. Non commutative symmetric functions of type B. PhD thesis, MIT, 2001.

[8] D. Garfinkle. On the classification of primitive ideals for complex classical Lie algebras, I. Compositio Mathematica, 75(2):135-169, 1990.

[9] D. Garfinkle. On the classification of primitive ideals for complex classical Lie algebras, II. Compositio Mathematica, 81(3):307-336, 1992.

[10] D. Garfinkle. On the classification of primitive ideals for complex classical Lie algebras, III. Compositio Mathematica, 88(2):187-234, 1993.

[11] A. Garsia and C. Reutenauer. A decomposition of Solomon's descent algebra. Advances in mathematics, 77:189-262, 1989.

[12] I. Gessel. Multipartite P-partitions and inner products of skew Schur functions. Contemporary Mathematics, 34:289-317, 1984.

[13] A. Kirillov, A. Lascoux, B. Leclerc, and J. Thibon. Séries génératrices pour les tableaux de dominos. C.R. Acad. Sci. Paris, 318(I):395-400, 1994.

[14] D. Knuth. Permutations, matrices and generalized young tableaux. Pacific J. Math., 34:709-727, 1970. 
[15] I. Macdonald. Symmetric functions and Hall polynomials. Oxford University Press, 1999.

[16] P. Norton. 0-Hecke algebras. J. Austral. Math. Soc. Ser A, 27:337-357, 1979.

[17] T. K. Petersen. A note on three types of quasisymmetric functions. The Electronic Journal of Combinatorics, 12:R61, 2005.

[18] S. Poirier. Cycle type and descent set in wreath products. Discrete Math., 180:315-343, 1998.

[19] L. Solomon. A Mackey formula in the group ring of a Coxeter group. Journal of Algebra, 41:255-264, 1976.

[20] R. Stanley. Enumerative combinatorics, volume 2. Cambridge University Press, 2001.

[21] D. Stanton and D. White. A Schensted algorithm for rim hook tableaux. Journal of Combinatorial Theory, Series A, 40:211-247, 1985.

[22] M. Taşkin. Plactic relations for r-domino tableaux. The Electronic Journal of Combinatorics, 19:P38, 2012.

[23] M. A. A. van Leeuwen. The Robinson-Schensted and Schützenberger algorithms, an elementary approach. The Electronic Journal of Combinatorics, 3(2):R15, 1996. 